\title{
Unilateral Upper Lung Field Pulmonary \\ Fibrosis Radiologically Consistent with Pleuroparenchymal Fibroelastosis after Thoracotomy: A New Disease Entity Related to Thoracotomy
}

\author{
Akimasa Sekine $^{a}$ Hiroaki Satoh $^{c}$ Tae Iwasawa $^{b}$ Kentaro Matsui $^{b}$ Eriko lkeya $^{a}$ \\ Satoshi Ikeda ${ }^{a}$ Hideaki Yamakawa $^{a}$ Ryo Okuda ${ }^{a}$ Hideya Kitamura ${ }^{a}$ \\ Takeshi Shinohara $^{\mathrm{a}}$ Tomohisa Baba $^{\mathrm{a}}$ Shigeru Komatsu ${ }^{\mathrm{a}}$ Terufumi Kato $^{\mathrm{a}}$ \\ Eri Hagiwara $^{a}$ Takashi Ogura $^{a}$ \\ Departments of ${ }^{\mathrm{a}}$ Respiratory Medicine and ${ }^{\mathrm{b}}$ Radiology, Kanagawa Cardiovascular and Respiratory Center, \\ Yokohama, and ${ }^{C}$ Department of Internal Medicine, Mito Medical Center, University of Tsukuba, Mito, Japan
}

\section{Keywords}

Apical cap · Pleuroparenchymal fibroelastosis .

Thoracotomy $\cdot$ Magnetic resonance imaging

\begin{abstract}
Background: Pleuroparenchymal fibroelastosis (PPFE) is a rare bilateral idiopathic interstitial pneumonia defined by pleural-parenchymal involvement. In clinical practice, we encountered patients with upper lung field pulmonary fibrosis (Upper-PF), which was radiologically consistent with PPFE, but apparently limited to the unilateral lung. Objectives: The purpose of the study was to clarify the clinical characteristics in those patients. Methods: We examined the medical records of all the consecutive patients from 2012 to 2016 to see whether there were patients having unilateral Upper-PF. Results: We found 6 patients with unilateral Upper-PF. The most common symptom was dyspnea, and all patients had a low body mass index and severe restrictive
\end{abstract}

(C) 2017 S. Karger AG, Basel

\section{KARGER}

E-Mail karger@karger.com

www.karger.com/res pulmonary impairment. Notably, all patients had a history of thoracotomy for resecting lung or esophageal cancer, and the lesions were limited to the operated side. Dynamic breathing chest MRI showed an impaired thoracic movement in the operated side. Serial chest CT from prethoracotomy to the first visit was obtained in 5 patients: before thoracotomy, only a slight apical cap, defined as a wedge- and triangle-shaped opacity with broad pleural contact, was observed only in the operated side, but progressed into the lesion after a median of 8.4 years following thoracotomy. After the first visit, the unilateral lesion rapidly deteriorated in all patients. Conclusions: Unilateral Upper-PF had some characteristics in common with PPFE. Because the lesion was limited to the operated side, unilateral Upper-PF would be a new disease entity related to thoracotomy. Our results indicate that thoracotomy impairs thoracic movement in the operated side and subsequently triggers unilateral Upper-PF development, especially in patients with an apical cap.

(c) 2017 S. Karger AG, Basel

Dr. Akimasa Sekine

Kanagawa Cardiovascular and Respiratory Center

Tomioka-Higashi 6-16-1, Kanazawa-ku

Yokohama 236-0051 (Japan)

E-Mail Akimasa.Sekine@gmail.com 


\section{Introduction}

Idiopathic pleuroparenchymal fibroelastosis (PPFE) is a rare form of bilateral interstitial pneumonia and characterized as marked pleural and parenchymal involvement predominantly in the upper lobe [1]. Radiologically, PPFE is defined as a presentation of pleural thickening with associated subpleural fibrosis $[2,3]$. In our clinical practice, we have encountered cases of upper lung field pulmonary fibrosis (Upper-PF) that are limited to the unilateral lung but are similar to PPFE in their radiological manifestations. We also took notice of the presence of apical caps, defined as a wedge- and triangle-shaped opacity with broad pleural contact in the apex of the lung in those patients [4]. We supposed that there would be a relationship between Upper-PF and the presence of apical cap. To clarify this relationship and the characteristics of unilateral Upper-PF, we performed a retrospective study.

\section{Method}

We examined the medical records of all consecutive patients who presented to our department from March 2012 to December 2016 to see whether there were patients displaying features of unilateral pleuroparenchymal disease. We gathered them and reviewed their clinical features.

Radiological findings of the unilateral lung with the lesion must be the same as those for "definite PPFE" or "consistent with PPFE." Patients who had apparent abnormal shadows in the contralateral lung on chest radiography were excluded. According to the previous report by Reddy et al. [2], "definite PPFE" was defined as demonstrating pleural thickening with associated subpleural fibrosis concentrated in the upper lobes, with involvement of the lower lobes being less marked or absent. "Consistent with PPFE" was defined as pleural thickening in the upper lobes with associated subpleural fibrosis present; however, the distribution of these changes is not concentrated in the upper lobes or the features of coexistent disease present elsewhere. Patients who were considered to have pulmonary aspergillosis and mycobacterial lung disease based on the results of sputum and serological examination were excluded. Patients were selected using medical charts and imaging studies on the basis of consensus of at least chest physicians or radiologists who had board certification of respirology or radiology.

Patients' characteristics including age, sex, smoking habit, previous history, and body mass index (BMI) were collected from medical records. In addition, the results of laboratory data includ-

Fig. 1. a MDT is shown in a' and b'. Chest wall motion is evaluated measuring the transvertical anteroposterior dimension of the upper and lower thoraces at mid-sagittal planes. The MACW was calculated as a' - c' and b' - d'. Respiratory motion of the diaphragm is also evaluated measuring the vertical length from the apex to each hemidiaphragm at a mid-coronal plane of breathing ing Krebs von den Lungen-6 (KL-6) and surfactant protein D (SP$\mathrm{D})$, chest high-resolution CT findings, lung perfusion scintigraphy, and pulmonary functional test were assessed. With regard to chest high-resolution CT, we took notice of the presence of an apical cap and the radiological change of the apical cap in the clinical course and, therefore, also evaluated serial CT scans before and after the time of the first visit. Besides, the motion of the diaphragm and chest wall was evaluated using dynamic breathing MRI.

\section{Dynamic Breathing MRI and Statistics}

All MRI studies were performed on a 1.5-T Achiva (Philips Medical Systems, Amsterdam, The Netherlands) using a 32-channel Torso coil. A balanced fast-field echo sequencing was used (echo time $1.1 \mathrm{~ms}$, repetition time $2.1 \mathrm{~ms}$, flip angle $50^{\circ}$, parallel imaging factor 2). The field of view was $450 \mathrm{~mm}$, and the matrix size was $256 \times 256$. The image slice thickness was $10 \mathrm{~mm}$, and acquisition time per image was $0.28 \mathrm{~s}$.

Coronal and sagittal imaging of the patient's thorax in the supine position was performed. Each patient was instructed to repeat breathing deeply from maximal inspiration to maximal expiration with as much effort as possible. Dynamic breathing MRI was performed at a fixed mid-coronal plane through the trachea and at two fixed mid-sagittal planes through the middle of each lung. Respiratory diaphragm and chest wall motion were evaluated by a cineloop view of the sequential MR images according to the paper reported by Suga et al. [5]. Respiratory diaphragm motions were evaluated measuring the maximal and minimal dimensions from the top of the apex to the diaphragm on a mid-coronal, and those of the transvertical anteroposterior dimension of the chest at the two fixed levels through the upper and lower thoraces (at $5 \mathrm{~cm}$ below the lung apex and at $5 \mathrm{~cm}$ above the diaphragm in the maximal inspiration) on mid-sagittal planes. The maximum anteroposterior diameter of the thorax (MDT) and the maximum amplitude of respiratory motions (the maximum dimension - the minimum dimension) of the chest walls (MACW) and diaphragm (MAD) were measured in each thorax as shown in Figure 1a. The parameters of MDT, MACW and MAD were compared between the right and left thorax, using the paired Student $t$ test, and $p$ values less than 0.05 were considered statistically significant. The software used for all analyses was JMP 10 software (SAS Institute, Cary, NC, USA). This study was approved by the Institutional Ethical Review Board (IRB: H27-25).

\section{Results}

\section{Patients' Characteristics}

Six patients were found to have the above-mentioned conditions (see the Methods). The patients' characteristics are described in Table 1 . The median age was 74.5
MRI. The MAD was calculated as e' - $\mathbf{f}$. b-d The details of the respiratory motion of the chest wall and diaphragm in case 1 (b), case 2 (c), and case $4(\mathbf{d})$. MDT, maximum anteroposterior diameter of the thorax; MACW, maximum amplitude of respiratory motions of the chest walls; MAD, maximum amplitude of respiratory motions of the diaphragm. 

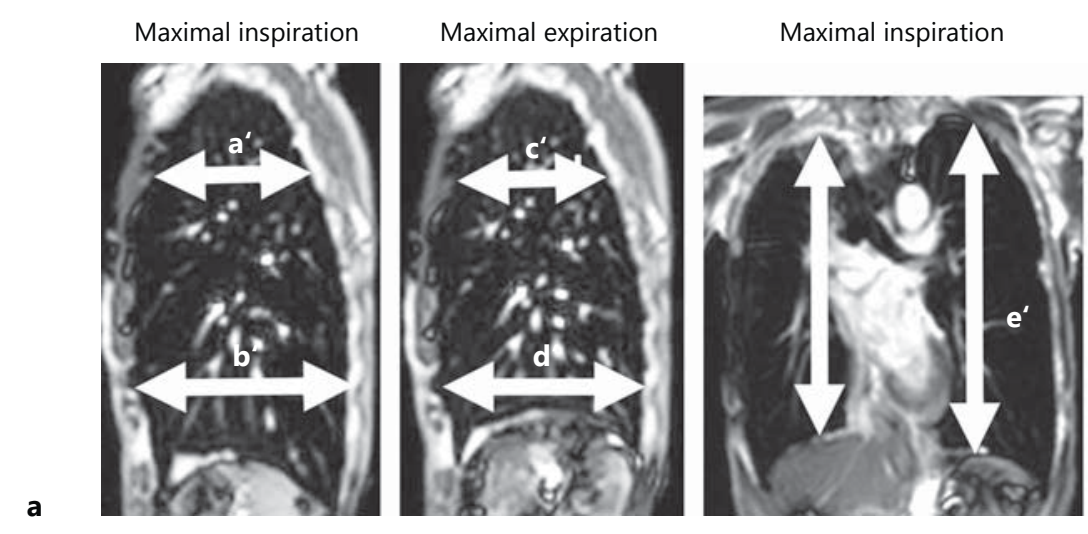

Maximal expiration

Chest wall motion right thorax

Chest wall motion left thorax
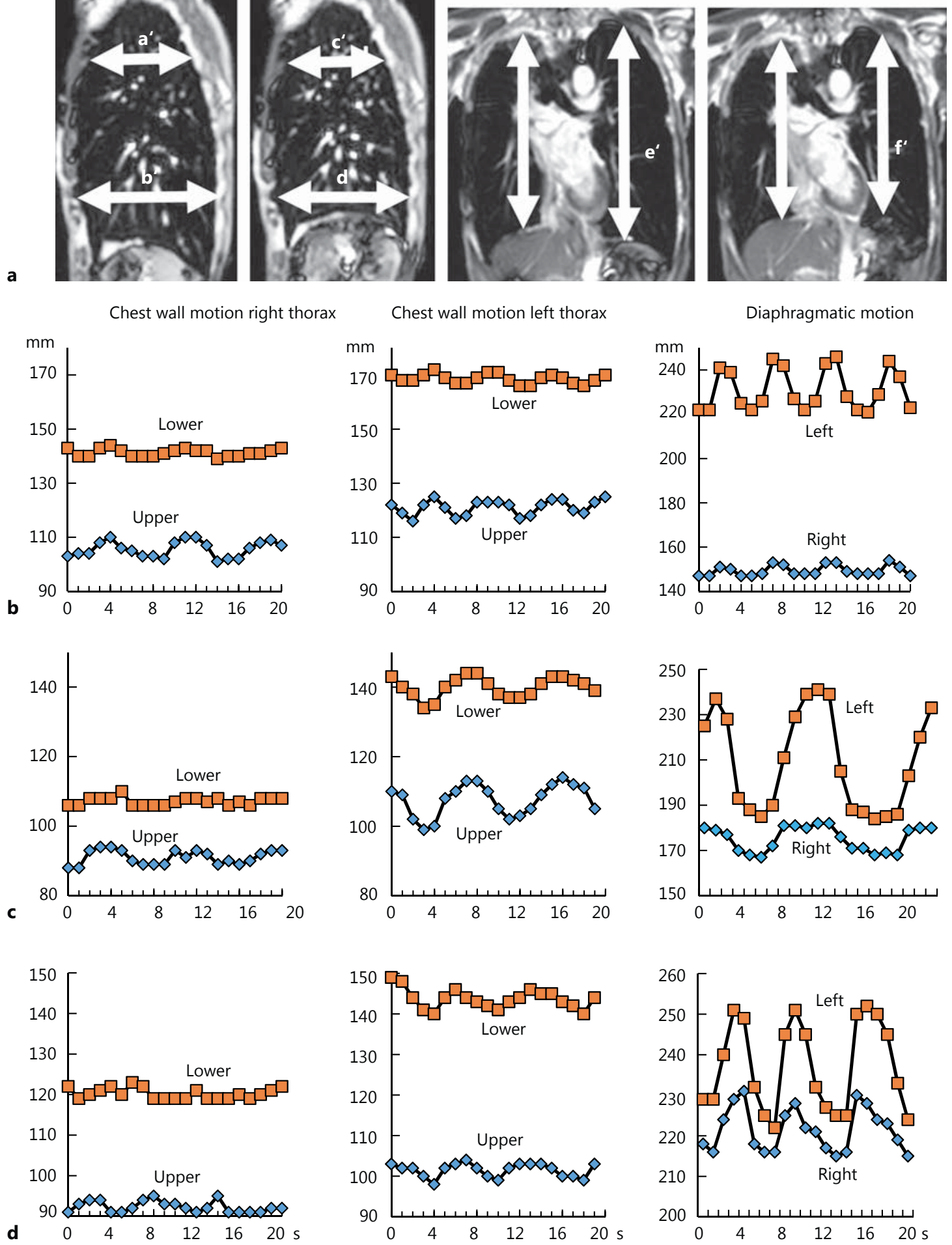
Table 1. Patient characteristics of 6 cases

\begin{tabular}{|c|c|c|c|c|c|c|c|c|c|c|}
\hline Case & $\begin{array}{l}\text { Age, } \\
\text { years/ } \\
\text { sex }\end{array}$ & $\begin{array}{l}\text { Chief } \\
\text { complaint }\end{array}$ & $\begin{array}{l}\text { Smoking } \\
\text { history } \\
\text { (pack-years) }\end{array}$ & $\begin{array}{l}\text { Previous } \\
\text { history }\end{array}$ & History of surgery & Resected lung & $\begin{array}{l}\text { Interval from } \\
\text { thoracic } \\
\text { surgery to first } \\
\text { visit, years }\end{array}$ & BMI & KL-6 & SP-D \\
\hline 1 & $70 / \mathrm{M}$ & Dyspnea & Former (23) & Lung ca, gastric ca & $\begin{array}{l}\text { Thoracotomy, } \\
\text { gastrectomy }\end{array}$ & Rt lower lobe & 11.8 & 18.2 & 342 & 145.2 \\
\hline 2 & $73 / \mathrm{M}$ & Rt back pain & Never & Lung ca & Thoracotomy & $\begin{array}{l}\text { Rt upper lobe } \\
\text { Rt middle lobe }\end{array}$ & 4.2 & 20.2 & 439 & 44.0 \\
\hline 3 & $78 / \mathrm{F}$ & $\begin{array}{l}\text { Dyspnea, cough, } \\
\text { sputum }\end{array}$ & Never & Lung ca & Thoracotomy & $\begin{array}{l}\text { Rt middle lobe } \\
\text { S6 }\end{array}$ & 14.3 & 19.6 & ne & ne \\
\hline 4 & $75 / \mathrm{M}$ & $\begin{array}{l}\text { Dyspnea, cough, } \\
\text { sputum }\end{array}$ & Former (5) & Esophageal ca & $\begin{array}{l}\text { Thoracotomy, } \\
\text { esophagectomy }\end{array}$ & & 2.8 & 19.1 & 359 & 316.8 \\
\hline 5 & $84 / \mathrm{M}$ & Cough & Former (36) & Esophageal ca & $\begin{array}{l}\text { Thoracotomy, } \\
\text { esophagectomy }\end{array}$ & & 10.5 & 18.7 & 581 & 221.1 \\
\hline 6 & $74 / \mathrm{F}$ & Dyspnea & Never & Lung ca & Minithoracotomy ${ }^{\mathrm{b}}$ & Rt lower lobe & 6.2 & 20.7 & 475 & 120.1 \\
\hline Median & 74.5 & & & & & & 8.4 & 19.4 & 439 & 145.2 \\
\hline
\end{tabular}

Rt, right; ca, carcinoma; KL-6, Krebs von den Lungen-6; SP-D, surfactant protein D; ne, not evaluated; BMI, body mass index. ${ }^{\text {a }}$ Partial resection. ${ }^{\mathrm{b}}$ Minithoracotomy with video assistance $(5 \mathrm{~cm}$ incision).

Table 2. Radiological findings of chest CT and lung perfusion scintigraphy, and results of pulmonary functional analysis

\begin{tabular}{|c|c|c|c|c|c|c|c|c|c|}
\hline \multirow[t]{2}{*}{ Case } & \multirow{2}{*}{$\begin{array}{l}\text { Lesion } \\
\text { location }\end{array}$} & \multicolumn{2}{|c|}{ Apical cap in the left lung } & \multicolumn{2}{|c|}{ Lung perfusion scintigraphy, \% } & \multicolumn{4}{|c|}{ Pulmonary functional analysis } \\
\hline & & first visit & $\begin{array}{l}\text { disease course } \\
\text { after first visit }\end{array}$ & right & left & $\% F V C$ & $\begin{array}{l}\text { FEV1, } \\
\%\end{array}$ & $\begin{array}{l}\text { \%RV/ } \\
\text { TLC }\end{array}$ & $\%$ DLCO \\
\hline 1 & Right & Present & Deteriorated & 12 & 88 & 34.1 & 98.4 & 175.4 & 165.3 \\
\hline 2 & Right & Present & Stable & 9 & 91 & 59.4 & 88.1 & ne & ne \\
\hline 3 & Right & Present & Stable & 27 & 73 & 74.8 & 70.6 & 131.1 & 115 \\
\hline 4 & Right & Present & Deteriorated & 20 & 80 & 46.9 & 97.2 & 145.5 & 137.4 \\
\hline 5 & Right & Present & Deteriorated & 34 & 66 & 64.2 & 91.8 & ne & ne \\
\hline \multirow[t]{2}{*}{6} & Right & Present & Stable & ne & ne & 75.9 & 69.3 & 97.5 & 89.8 \\
\hline & Median & & & 20 & 80 & 61.8 & 90.0 & 138.3 & 126.2 \\
\hline
\end{tabular}

CT, computed tomography; ne, not evaluated; FVC, forced vital capacity; FEV1, forced expiratory volume in $1 \mathrm{~s}$; RV, residual volume; DLCO, diffusing lung capacity for carbon monoxide.

years. Four of the 6 patients were male and 3 patients were former smokers. The most common symptom was dyspnea, and all patients had a history of surgical treatment for various cancers: lung cancer in 4 patients, esophageal cancer in 2 patients, and gastric cancer in 1 patient. Last surgical treatment was for lung cancer in 3 patients, esophageal cancer in 2 patients, and gastric cancer in 1 patient. Of note, all patients had undergone right thoracotomy for resecting lung or esophageal cancer. Surgical lung resection was performed in 4 patients: right lower lobectomy in 2 patients, right upper lobectomy and mid- dle lobe partial resection in 1 patient, and right middle lobectomy and S6 segmentectomy in the remaining patient. The median interval from thoracotomy to the first visit to our hospital was 8.4 years. In all patients, BMI was low, with a median of 19.4. Regarding markers for interstitial lung disease, KL-6 was within the normal range whereas SP-D was elevated in almost all patients. The presence of antinuclear antibody was assessed in all patients, but it was positive in only 1 patient. This patient did not display features suggestive of an underlying connective tissue disease. 
a

Fig. 2. a No abnormal shadow was detected 1 year after surgical resection of the right upper lobectomy and middle lobe partial resection. b A subpleural and parenchymal lesion developed in the remaining right lung 4 years after surgical lung resection.

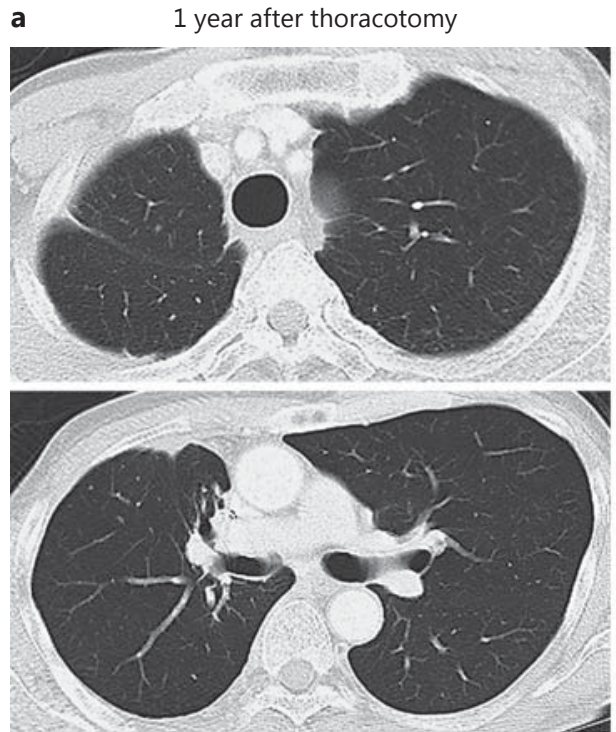

b $\quad 4$ years after thoracotomy
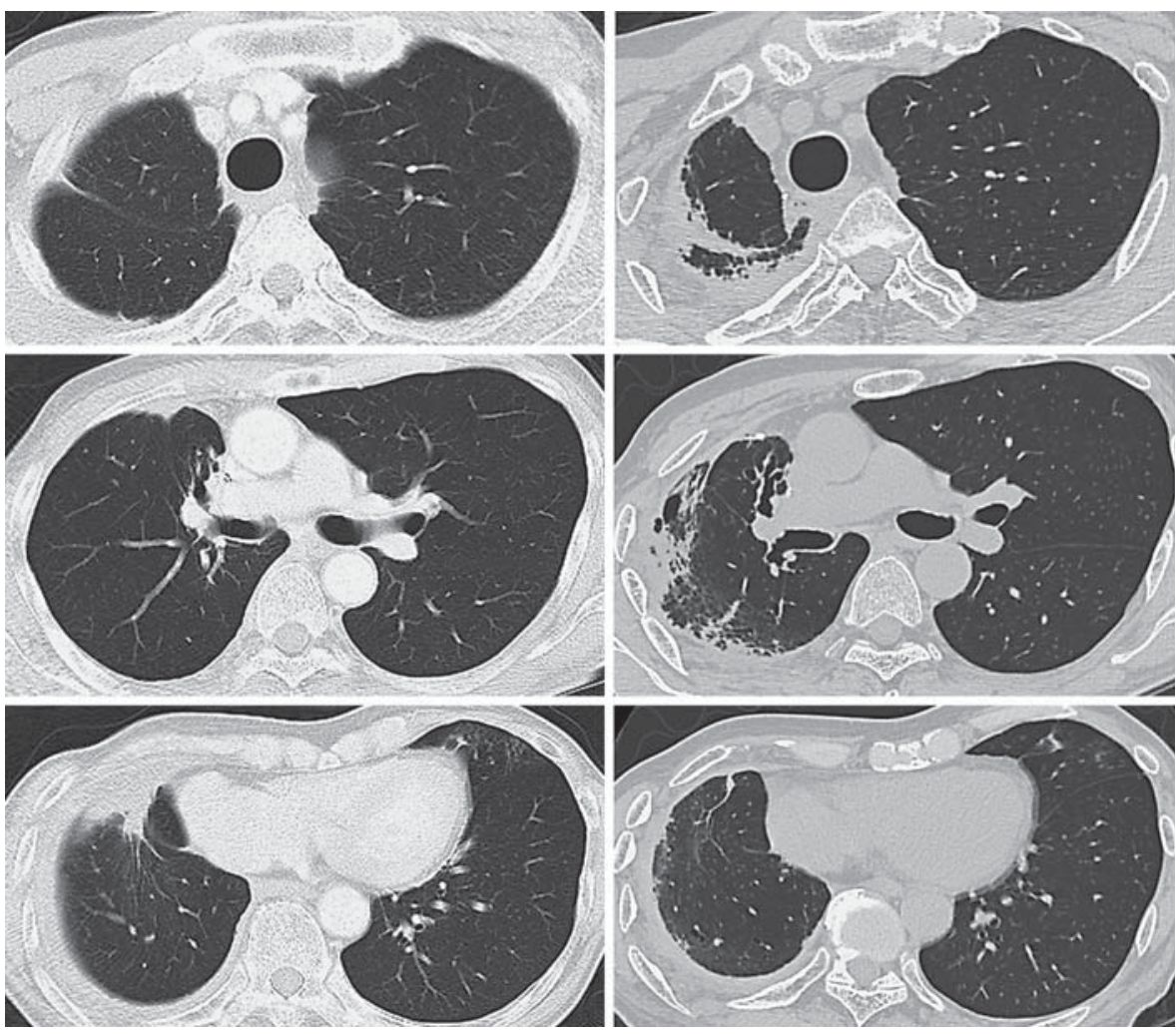

Serial Chest CT Finding and the Relationship of Apical Cap and Pleuroparenchymal Involvement (Table 2)

All patients were evaluated with chest high-resolution $\mathrm{CT}$ on the first visit. Importantly, in all patients, pleuroparenchymal involvement was present in the right side (operated side). Five patients had pleural thickening and subpleural fibrosis limited to the right upper lobe, which radiologically met the criteria of definite PPFE. In the remaining patient with a history of right upper lobectomy and middle-lobe partial resection, pleural thickening and subpleural fibrosis was present in the right middle and lower lobes, which met the criteria of consistent with PPFE (Fig. 2). With regard to the left lung (nonoperated side), only a pulmonary apical cap was present in all patients, although the degree was slight. The previous CT before thoracotomy was obtained in 5 patients. Notably, all of them had a right apical cap (operated side), which apparently deteriorated and progressed into the lesion by the time of the first visit to our hospital. After the first visit, the right lesion in the operated side rapidly deteriorated in all patients. However, with respect to the radiological course of the left apical cap (nonoperated side), the

Unilateral Upper-PF Radiologically

Consistent with PPFE after Thoracotomy progression was observed only in the 3 patients with a history of gastric or esophageal resection, whereas the remaining 3 patients without those histories presented stable disease. In Figure 3, we present the radiological course of case 4 with a history of thoracotomy for esophagectomy. Before right thoracotomy, a slight apical cap was only present in the operated side (Fig. 3a), but it deteriorated and resulted in pleural-parenchymal involvement with an emergence of a left apical cap (Fig. 3b). During the clinical course, the right lesion and left apical cap apparently aggravated (Fig. 3c).

\section{Lung Perfusion Scintigraphy and Pulmonary Function Analysis}

In 5 of 6 patients, lung perfusion scintigraphy was performed and showed a drastic reduction of the right perfusion rate, which ranged from 9 to $34 \%$ with a median of $20 \%$. In all evaluated patients, reduced perfusion was evident in the right upper lung field.

With regard to pulmonary function analysis, all patients presented a remarkable restrictive dysfunction with a normal range of forced expiratory volume in $1 \mathrm{~s}$ (FEV1)/ forced vital capacity (FVC). \%FVC was low and ranged 
a
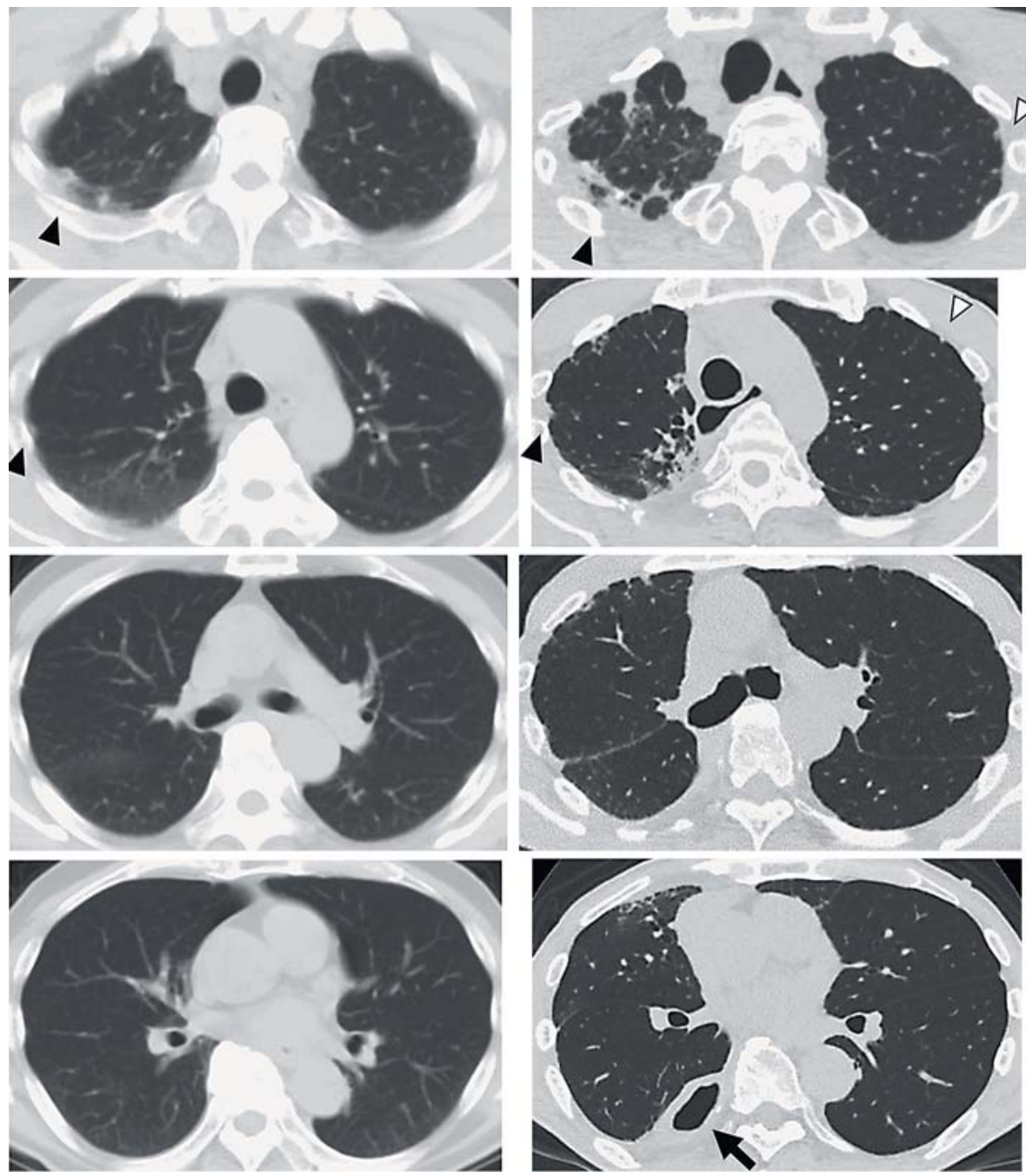

C
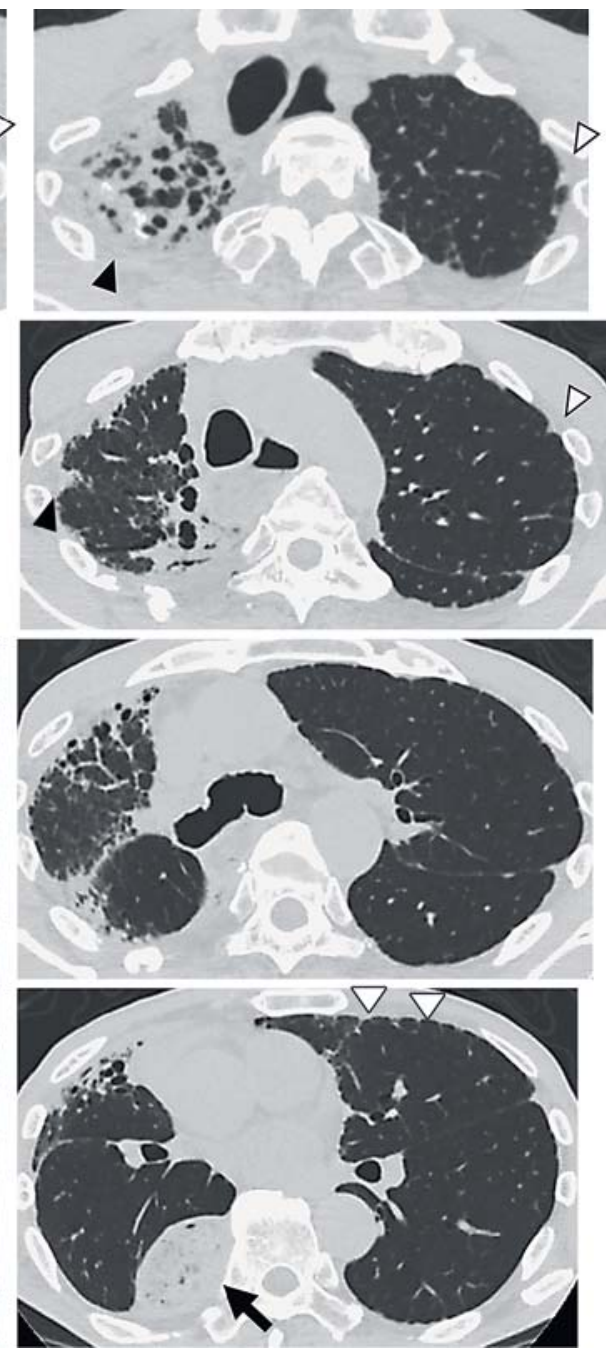

Fig. 3. a Before thoracotomy for esophagectomy; chest CT in February 2011 demonstrating normal lung with a subtle right pulmonary apical cap (black arrowhead). b Chest CT on the first visit (in December 2013) revealing a slight subpleural lesion in the right lung (black arrowhead) with a slight pulmonary apical cap in the left lung (white arrowhead). In the dilated uplifted stomach, an airfluid interface was visible (arrow). c Chest CT in February 2015 showing a progression of the right lung lesion and left pulmonary apical cap (arrowheads) with a full stomach (arrow). from 34.1 to $75.9 \%$, with a median of $61.8 \%$. Almost all patients presented normal FEV1\% with a median of $90.0 \%$. On the other hand, percent residual volume/total lung capacity and percent diffusing capacity of the lung carbon monoxide were greater, with medians of 138.3 and $126.2 \%$, respectively.

\section{Dynamic Breathing MRI}

In 5 patients, dynamic breathing MRI was performed to analyze the movement of the diaphragm and chest wall as shown in Figure 1 and Table 3. Right MDT was signifi- cantly smaller than left MDT at both upper and lower thoracic levels $(p=0.011,0.045)$, indicating that the patients had a flattened chest in the operated right side. With regard to chest wall movement, right MACW was smaller compared to left MACW at both the upper and lower thoracic levels ( $p=0.028,0.047)$. Regarding diaphragm movement, MAD was also smaller in the right thorax than in the left thorax ( $p=0.028)$. These results showed an impaired movement of the chest wall and diaphragm in the operated right side. The details of respiratory motion of cases 1, 2, and 4 are presented in Figure 1b-d. 
Fig. 4. a Chest radiograph showing a lesion in the right upper lung field. b Chest CT demonstrating subpleural parenchymal involvement in the right upper lobe, with a slight pulmonary apical cap in left upper lobe (arrowhead).

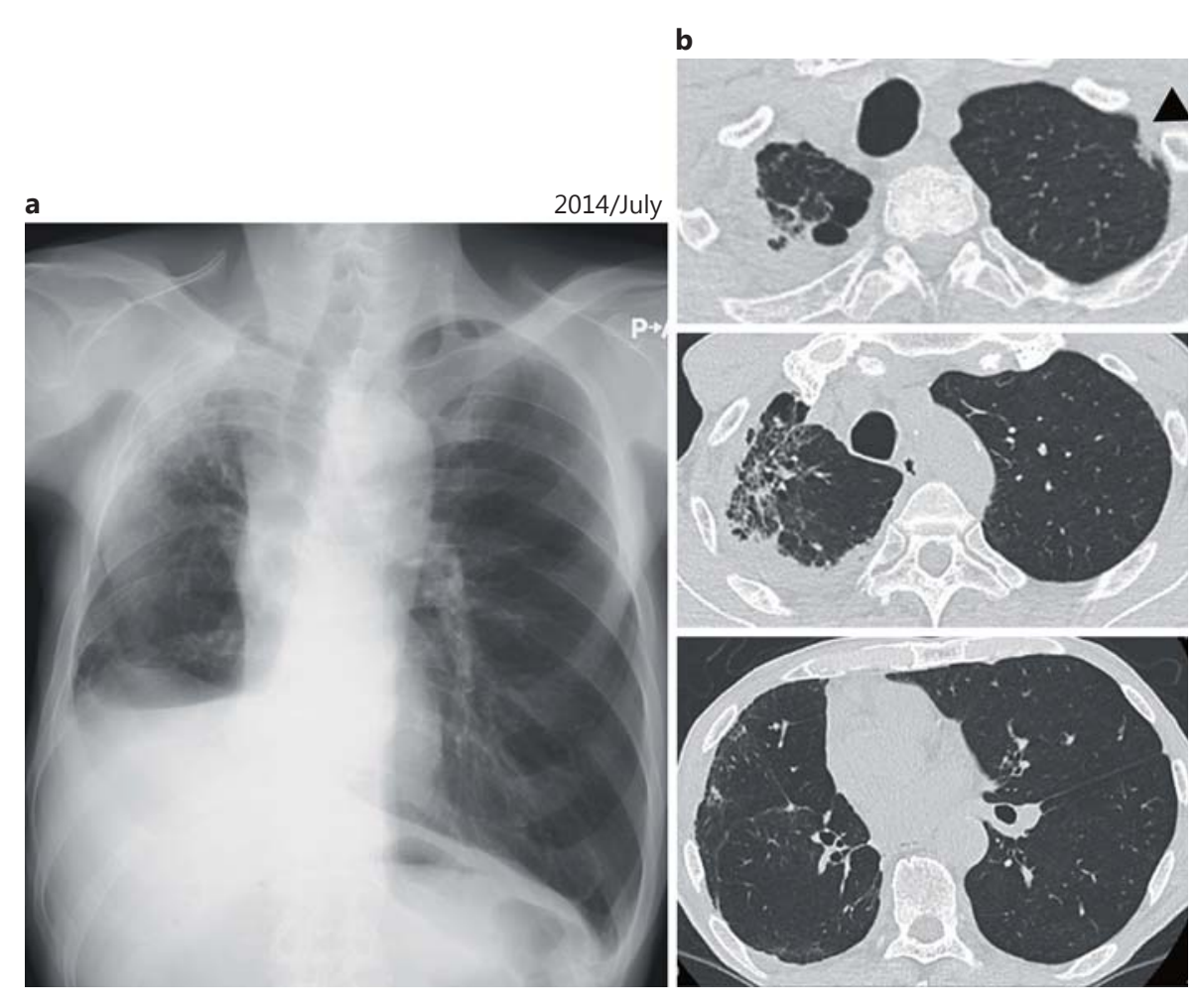

b

Table 3. Dynamic breathing magnetic resonance imaging

\begin{tabular}{|c|c|c|c|c|c|c|c|c|c|c|}
\hline \multirow[t]{3}{*}{ Case } & \multicolumn{4}{|c|}{ MDT, mm } & \multicolumn{4}{|c|}{$\mathrm{MACW}, \mathrm{mm}$} & \multicolumn{2}{|c|}{$\mathrm{MAD}, \mathrm{mm}$} \\
\hline & \multicolumn{2}{|l|}{ upper } & \multicolumn{2}{|l|}{ lower } & \multicolumn{2}{|l|}{ upper } & \multicolumn{2}{|l|}{ lower } & \multirow[b]{2}{*}{ right } & \multirow[b]{2}{*}{ left } \\
\hline & right & left & right & left & right & left & right & left & & \\
\hline 1 & 110 & 144 & 125 & 172 & 9 & 9 & 5 & 6 & 7 & 25 \\
\hline 2 & 94 & 114 & 110 & 144 & 6 & 15 & 4 & 10 & 15 & 57 \\
\hline 3 & 142 & 166 & 146 & 175 & 19 & 25 & 15 & 18 & 42 & 58 \\
\hline 4 & 95 & 104 & 123 & 149 & 4 & 6 & 4 & 9 & 16 & 30 \\
\hline 5 & 114 & 126 & 157 & 150 & 6 & 15 & 4 & 9 & 22 & 22 \\
\hline 6 & ne & ne & ne & ne & ne & ne & ne & ne & ne & ne \\
\hline Mean \pm SEM & $111 \pm 9$ & $131 \pm 11$ & $132 \pm 8$ & $158 \pm 6$ & $8.8 \pm 2.7$ & $14 \pm 3.3$ & $6.4 \pm 2.2$ & $10.4 \pm 2.0$ & $20.4 \pm 5.9$ & $38.4 \pm 7.9$ \\
\hline Median & 110 & 126 & 125 & 150 & 6 & 15 & 4 & 9 & 16 & 30 \\
\hline
\end{tabular}

MDT, maximum anteroposterior diameter of the thorax; MACW, maximum amplitude of respiratory motions of chest walls; MAD, maximum amplitude of respiratory motions of the diaphragm; ne, not evaluated.

\section{Case Presentation (Case 1)}

In July 2014, a 70-year-old man visited our hospital complaining of gradually deteriorating dyspnea on exertion. He had a previous history of right lower lobectomy for lung adenocarcinoma in September 2002 and distal gastrectomy for gastric cancer in November 2011. After gastrectomy, his body weight decreased by $10 \mathrm{~kg}$ over 3 years. Chest radiography on the first visit to our hospital showed a volume loss in the right lung, and chest highresolution CT demonstrated pleural and parenchymal involvement in the right upper lobe with a subtle apical cap in the left lung (Fig. 4). Notably, although there was no 
a

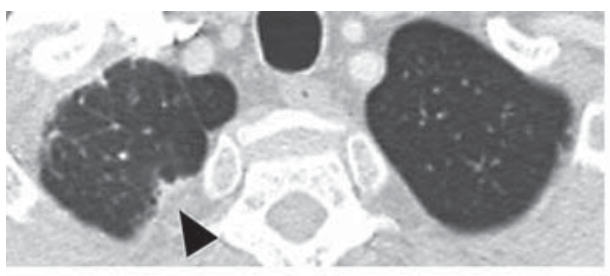

b

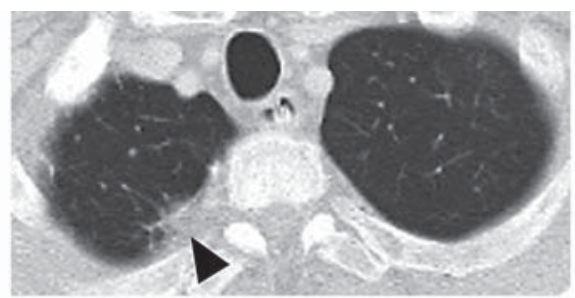

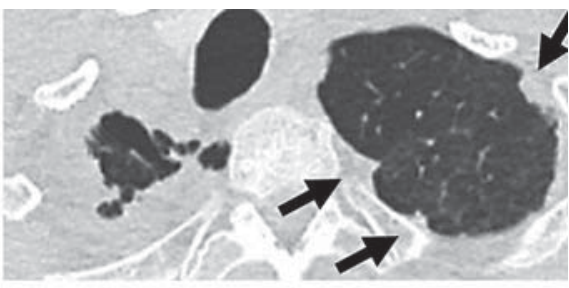
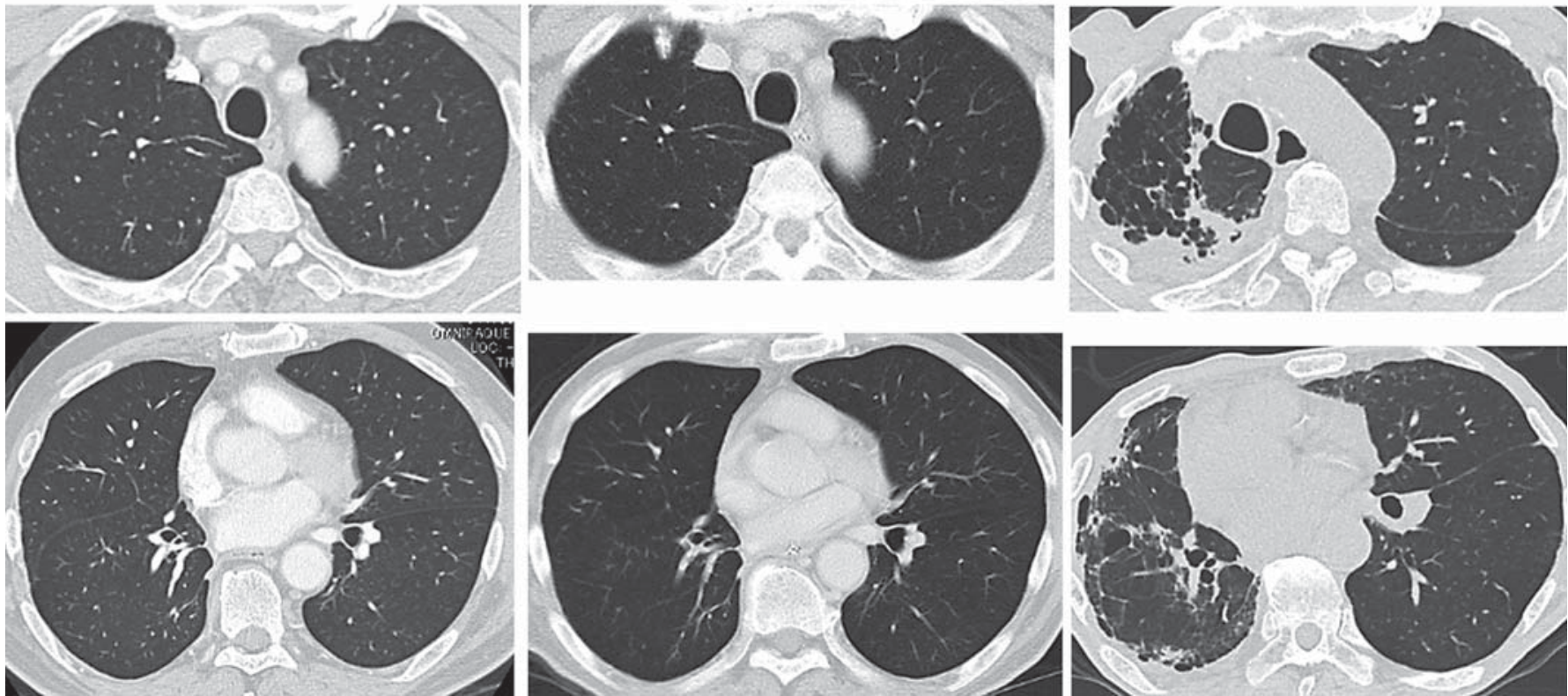

Fig. 5. Serial chest CTs between October 2007 (a) and January 2012 (b) demonstrating only a slight pulmonary apical cap in the right upper lobe (arrowhead) with no apparent change. In February

2016, a subpleural parenchymal involvement with an emphysematous change developed in the right lung and the left pulmonary apical cap (arrow) apparently aggravated (c).

apparent difference of chest CT findings between 2007 and 2012 (Fig. 5a, b), serial chest CT showed that the right apical cap rapidly deteriorated and progressed into the lesion after gastrectomy (Fig. 4b). A pulmonary function test on the first visit showed a decreased \%FVC of $34.3 \%$ with an elevated percent residual volume/total lung capacity of $175.4 \%$, whereas $\%$ FVC in November 2011 was $69.2 \%$. In lung perfusion scintigraphy, the perfusion rate of the right lung was extremely low at $12 \%$. Dynamic breathing MRI showed that the right MDT and MAD were smaller compared to the left ones, whereas the upper and lower MACW was equal on both sides of the chest wall (Fig. 1b). Because the arterial blood gas analysis showed hypercapnia, we initiated noninvasive positive pressure ventilation and rehabilitation to improve right thoracic movement, resulting in an increase in FVC from 1.28 to $1.53 \mathrm{~L}$ over a week. With nutrition therapy, noninvasive positive pressure ventilation, and pulmonary re- habilitation, the patient presented a stable disease course without body weight loss until July 2015 . However, a subpleural emphysematous-like change appeared in the right upper lobe, and the left apical cap apparently deteriorated in February 2016 (Fig. 5c).

\section{Discussion}

We reported on 6 consecutive cases of unilateral Upper-PF that radiologically met the criterion "definite PPFE" or "consistent with PPFE." To date, PPFE patients have been reported to present a low BMI with a flattened chest and a restrictive pulmonary impairment, which was observed in all our patients [6-9]. These results indicate that one of the differences between our patients and patients with PPFE was whether a lesion existed in the unilateral lung or bilateral lungs. The lesion was apparently 
Fig. 6. Schema of the possible mechanism of development of unilateral upper lung field pulmonary fibrosis (Upper-PF). GERD, Gastroesophageal reflux disease.

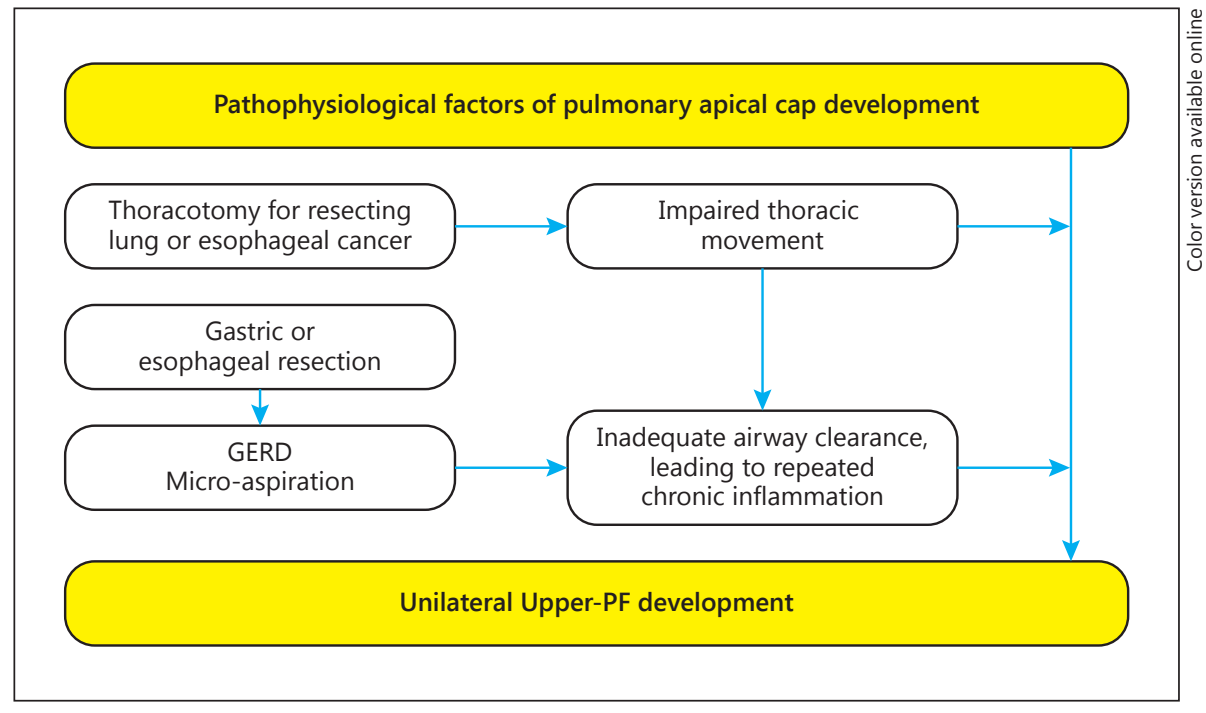

located in the operated side and therefore, unilateral Upper-PF would be a new disease entity related to thoracotomy. Of note, although unilateral Upper-PF developed in the upper lobe in 5 of 6 patients, the remaining patient with a history of upper lobectomy and middle lobe partial resection developed unilateral Upper-PF in the middle and lower lobes (case 2, Fig. 2). These results indicate that unilateral Upper-PF development is correlated with upper lung location, not with upper lobe. Although the mechanism of unilateral Upper-PF development remains unclear, our results suggest the following two possibilities.

Firstly, thoracotomy could cause unilateral Upper-PF, which would arise from impaired thoracic movement in the operated side, especially in patients with an apical cap. In fact, all patients had a history of right thoracotomy, and the lesions were located on the operated side. Importantly, 2 of them had an esophageal cancer, and therefore, lung resection was not performed (cases 4 and 5). These results strongly indicate that thoracotomy itself is an important factor for developing unilateral Upper-PF. Generally, thoracotomy causes a restrictive pulmonary impairment compared to complete video-assisted thoracic surgery, because ribs, the latissimus dorsi, and the serratus anterior muscles are dissected $[10,11]$. Indeed, all patients in this study presented a restrictive pulmonary impairment, and dynamic breathing MRI revealed an impaired movement of the chest wall and diaphragm in the operated side. These results suggest that thoracotomy has a substantial negative impact on thoracic movement in the operated side. However, we believe that impaired tho-

Unilateral Upper-PF Radiologically Consistent with PPFE after Thoracotomy racic movement alone would not cause unilateral Upper$\mathrm{PF}$, because the majority of patients treated with thoracotomy do not develop unilateral Upper-PF. The important finding in the present study is that all radiologically evaluated patients before thoracotomy had an apical cap in the operated side, which apparently deteriorated and progressed into the lesion during their clinical courses. These results imply that the presence of an apical cap in the operated side is particularly important for unilateral Upper-PF development. More recently, apical cap, which is pathologically identical to PPFE, has been reported to be potentially caused by relative ischemia in the upper lobe and low-grade inflammation in the lung parenchyma $[2,12]$. Besides, relative ischemia in the upper lobe can impair the resolution of inflammation, which would also facilitate the development of apical cap and PPFE $[4,13$, 14]. Taking those reports into consideration, together with our results, the patients with an apical cap would have a potential pathophysiological factor change of relative ischemia and low-grade inflammation to some extent, and thoracotomy would strongly affect those patients.

Secondly, gastroesophageal resection may partially contribute to pulmonary fibrosis development. Indeed, in case 1, unilateral Upper-PF developed soon after gastrectomy, although both lungs remained normal for 7 years after thoracotomy (Fig. 5a, b). Therefore, mechanisms other than an impaired thoracic movement should also contribute to the development of unilateral Upper-PF. We believe that the clinical courses of an apical cap in the nonoperated side, which was observed during the first visit in 
all patients, is important to clarify those mechanisms. Of note, apical caps in the nonoperated side apparently aggravated in the 3 patients with a history of gastrectomy or esophagectomy during their clinical course (cases 1, 4, and 5). These results indicate that gastroesophagectomy itself potentially aggravates the apical cap. Considering the previously mentioned reports that apical cap development was potentially correlated with low-grade inflammation $[2,12]$, we believe that our 3 patients with a history of gastroesophageal resection potentially had a mechanism of microaspiration, which resulted in repeated low-grade inflammation. Generally, gastroesophageal reflux disease (GERD) and delayed gastric emptying easily develop in patients with a history of gastrectomy or esophagectomy [15-17] and can cause aspiration pneumonia [18]. In fact, 2 patients treated with esophagectomy (cases 4 and 5) had an apparent air-fluid level in the remaining esophagus and intrathoracic stomach (Fig. 3b, c), suggesting that GERD and microaspiration frequently occur. Importantly, many recent studies have shown that microaspiration following GERD can cause interstitial lung disease $[19,20]$, which is predominant in the right lung as observed in our cases [21]. In addition, GERD-related microaspiration has been reported to potentially cause upper lobe dominant interstitial lung disease [22]. Probably, restrictive thoracic movement in the operated side would also contribute to aggravate the impaired airway clearance following microaspiration. The schema of this hypothesis of possible mechanisms for unilateral Upper-PF development is shown in Figure 6.

Despite these important results, our study had several limitations. Firstly, this was a small-sized retrospective study at a single institution. Secondly, it remains unclear how often unilateral Upper-PF actually developed in patients with apical cap, who underwent thoracotomy, be- cause only 2 patients who underwent thoracotomy were not treated in our hospital. Thirdly, no pathological specimens could be obtained, because the pulmonary functional results of the patients were not good enough to perform surgical lung biopsy in all patients. Therefore, it would be important in future research to investigate whether the pathological findings in unilateral upper-PF are same as those in PPFE.

In conclusion, we have reported on 6 consecutive cases of unilateral Upper-PF, which had some characteristics commonly observed in patients with PPFE. Because the lesion was limited to the operated side, unilateral Upper$\mathrm{PF}$ would be a new disease entity related to thoracotomy. Although the mechanism of unilateral Upper-PF remains unclear, thoracotomy impairs thoracic movement in the operated side and subsequently would trigger unilateral Upper-PF development, especially in patients with an apical cap. Accumulation of knowledge about unilateral Upper-PF will help clarify the mechanisms of PPFE development.

\section{Acknowledgments}

A.S. was the primary investigator and had full access to all the data in the study and takes responsibility for the integrity of the data and accuracy of the analysis. A.S., K.M., T.I., E.I., T.S., T.B., T.K., and T.O. were involved in data generation analysis. A.S. and H.S. were involved in drafting the manuscript, and K.M. and T.I. were responsible for evaluating radiological examination. All authors were responsible for the critical revision of the manuscript and approved the final manuscript.

\section{Financial Disclosure and Conflicts of Interest}

The authors declare that there are no conflicts of interest related to this article.
References

440
1 Frankel SK, Cool CD, Lynch DA, Brown KK: Idiopathic pleuroparenchymal fibroelastosis: description of a novel clinicopathologic entity. Chest 2004;126:2007-2013.

2 Reddy TL, Tominaga M, Hansell DM, von der Thusen J, Rassl D, Parfrey H, et al: Pleuroparenchymal fibroelastosis: a spectrum of histopathological and imaging phenotypes. Eur Respir J 2012;40:377-385.

3 Travis WD, Costabel U, Hansell DM, King TE Jr, Lynch DA, Nicholson AG, et al: An official American Thoracic Society/European Respiratory Society statement: update of the international multidisciplinary classification of the idiopathic interstitial pneumo- nias. Am J Respir Crit Care Med 2013;188: 733-748.

4 Lagstein A: Pulmonary apical cap - what's old is new again. Arch Pathol Lab Med 2015;139: 1258-1262.

5 Suga K, Tsukuda T, Awaya H, Takano K, Koike S, Matsunaga N, et al: Impaired respiratory mechanics in pulmonary emphysema: evaluation with dynamic breathing MRI. J Magn Reson Imaging 1999;10:510-520.

6 Watanabe K, Nagata N, Kitasato Y, Wakamatsu K, Nabeshima K, Harada T, et al: Rapid decrease in forced vital capacity in patients with idiopathic pulmonary upper lobe fibrosis. Respir Investig 2012;50:88-97. 
7 Watanabe S, Waseda Y, Takato H, Matsunuma R, Johkoh T, Egashira R, et al: Pleuroparenchymal fibroelastosis: distinct pulmonary physiological features in nine patients. Respir Investig 2015;53:149-155.

8 Harada T, Yoshida Y, Kitasato Y, Tsuruta N, Wakamatsu K, Hirota T, et al: The thoracic cage becomes flattened in the progression of pleuroparenchymal fibroelastosis. Eur Respir Rev 2014;23:263-266.

9 Watanabe K: Pleuroparenchymal fibroelastosis: its clinical characteristics. Curr Respir Med Rev 2013;9:299-237.

10 Kaseda S, Aoki T, Hangai N, Shimizu K: Better pulmonary function and prognosis with video-assisted thoracic surgery than with thoracotomy. Ann Thorac Surg 2000;70:16441646.

11 Tajiri M, Maehara T, Nakayama H, Sakamoto $\mathrm{K}$ : Decreased invasiveness via two methods of thoracoscopic lobectomy for lung cancer, compared with open thoracotomy. Respirology 2007;12:207-211.
12 Hirota T, Yoshida Y, Kitasato Y, Yoshimi M, Koga T, Tsuruta N, et al: Histological evolution of pleuroparenchymal fibroelastosis. Histopathology 2015;66:545-554.

13 Butler C 2nd, Kleinerman J: The pulmonary apical cap. Am J Pathol 1970;60:205-216.

14 Yousem SA: Pulmonary apical cap: a distinctive but poorly recognized lesion in pulmonary surgical pathology. Am J Surg Pathol 2001;25:679-683.

15 Haga N, Mochiki E, Nakabayashi T, Suzuki T, Asao T, Kuwano H: Esophageal manometric changes and gastroesophageal reflux symptoms after distal gastrectomy for gastric cancer. Hepatogastroenterology 2005;52:310313.

16 Marumo K, Homma S, Fukuchi Y: Postgastrectomy aspiration pneumonia. Chest 1995; 107:453-456.

17 Poghosyan T, Gaujoux S, Chirica M, MunozBongrand N, Sarfati E, Cattan P: Functional disorders and quality of life after esophagectomy and gastric tube reconstruction for cancer. J Visc Surg 2011;148:e327-e335.
18 Lerut TE, van Lanschot JJ: Chronic symptoms after subtotal or partial oesophagectomy: diagnosis and treatment. Best Pract Res Clin Gastroenterol 2004;18:901-915.

19 Raghu G, Freudenberger TD, Yang S, Curtis JR, Spada C, Hayes J, et al: High prevalence of abnormal acid gastro-oesophageal reflux in idiopathic pulmonary fibrosis. Eur Respir J 2006;27:136-142.

20 Tobin RW, Pope CE 2nd, Pellegrini CA, Emond MJ, Sillery J, Raghu G: Increased prevalence of gastroesophageal reflux in patients with idiopathic pulmonary fibrosis. Am J Respir Crit Care Med 1998;158:1804-1808.

21 Tcherakian C, Cottin V, Brillet PY, Freynet O, Naggara N, Carton Z, et al: Progression of idiopathic pulmonary fibrosis: lessons from asymmetrical disease. Thorax 2011;66:226231.

22 Cardasis JJ, MacMahon H, Husain AN: The spectrum of lung disease due to chronic occult aspiration. Ann Am Thorac Soc 2014;11: 865-873.
Unilateral Upper-PF Radiologically Consistent with PPFE after Thoracotomy
Respiration 2017;94:431-441 DOI: $10.1159 / 000479331$ 